\title{
The Role of GOLPH3L in the Prognosis and NACT response in Cervical Cancer
}

\author{
Yanling Feng $1^{*}$, Fan $\mathrm{He}^{2 *}$, Shumei Yan ${ }^{3^{*}}$, He Huang ${ }^{1}$, Qidan Huang${ }^{1}$, Ting Deng ${ }^{1}$, Huini Wu ${ }^{4}$, Bei Gao ${ }^{5}$, \\ Jihong Liu ${ }^{1 凶}$
}

1. Department of Gynecologic Oncology, Sun Yat-sen University Cancer Center; State Key Laboratory of Oncology in South China; Collaborative Innovation Center for Cancer Medicine, Guangzhou 510080, China.

2. Department of Molecular Oncology, H. Lee Moffitt Cancer Center \& Research Institute, Tampa, FL 33612, USA.

3. Department of Pathology, Sun Yat-Sen University Cancer Center; State Key Laboratory of Oncology in South China; Collaborative Innovation Center for Cancer Medicine, Guangzhou 510060, China.

4. Department of Biology, University of Illinois at Chicago, Chicago, IL 60607, United States.

5. State Key Laboratory of Ophthalmology, Zhongshan Ophthalmic Center, Sun Yat-sen University, Guangzhou, China.

*These authors contributed equally to this work and share first authorship.

$\triangle$ Corresponding author: Jihong Liu, Department of Gynecologic Oncology, Sun Yat-sen University Cancer Center; State Key Laboratory of Oncology in South China; Collaborative Innovation Center for Cancer Medicine, Guangzhou 510060, P.R. China. Email: liujh@sysucc.org.cn; Tel: +86- 20-87343105.

(C) Ivyspring International Publisher. This is an open access article distributed under the terms of the Creative Commons Attribution (CC BY-NC) license (https://creativecommons.org/licenses/by-nc/4.0/). See http://ivyspring.com/terms for full terms and conditions.

Received: 2016.08.03; Accepted: 2016.10.29; Published: 2017.02.10

\begin{abstract}
Background: We previously reported GOLPH3L is a novel oncogene associated with ovarian cancer. The role of GOLPH3L in cervical cancer and its cellular functions has not been determined. This study investigated clinical significance of GOLPH3L and potential proteins and pathways associated with GOLPH3L in cervical squamous cell carcinoma.

Methods: Immunohistochemistry and western blot were used to examine the expression of GOLPH3L in cervical squamous cell carcinoma tissue specimens and adjacent non-cancerous tissues. The clinical and prognostic significance of GOLPH3L expression was statistically analyzed. Cell proliferation rate, cell cycle progression, apoptosis and cisplatin response in GOLPH3L silenced SiHa and HeLa cells were also examined. Phospho-antibody array was used to identify changes in protein phosphorylation and the corresponding signaling pathways associated with these changes.

Results: GOLPH3L overexpressed in cervical cancer tissue specimens compared with normal adjacent non-cancerous tissues. Increased GOLPH3L expression was associated with FIGO staging $(\mathrm{P}=0.033)$, cervical stromal invasion $(P=0.037)$, cervical canal stromal invasion $(P=0.027)$, lymph node metastasis $(P=0.016)$ and positive surgical margins $(P=0.015)$. Patients with lower expression of GOLPH3L demonstrated longer progression-free survival and overall survival compared with those with higher expression. The tissue samples from patients who poorly responded to neoadjuvant chemotherapy (NACT) exhibited increased GOLPH3L expression levels compared with tissue samples from patients who achieved a pathologic complete response (pCR). Patients with lower GOLPH3L expression level, poorer tumor differentiation, shorter NACT treatment intervals and smaller tumor sizes were more likely to achieve a PCR after NACT. Knockdown GOLPH3L in cells was associated with an induction of cell cycle arrest, increased apoptosis and cisplatin sensitivity, and a reduction in cellular viability. Phospho-antibody array suggested GOLPH3L plays a role in mediating cell cycle arrest.

Conclusions: This study provides a potential biomarker for predicting prognosis and NACT response in patients with cervical squamous cell carcinoma. The functional role of GOLPH3L in cervical cancer merits further investigation.
\end{abstract}

Key words: GOLPH3L; cervical cancer; NACT; cell cycle arrest.

\section{Introduction}

Cervical cancer is the fourth leading cause of cancer-related deaths in females worldwide. Each year, 530,000 new cases of cervical cancer are diagnosed worldwide, accounting for approximately 
$9 \%$ of new cases of cancer diagnosed in women each year. The overall five-year survival rate associated with all stages of cervical cancer is $68 \%$. However, the five-year survival rate in patients with distal metastases is only $16 \%$ [1]. Currently, the standard treatment approaches for cervical cancer include chemotherapy, radiology and surgery. In patients with locally advanced cervical cancer, neoadjuvant chemotherapy (NACT) has recently been adopted to reduce tumor size and to eliminate micrometastasis prior to surgery [2, 3]. However, biomarkers that accurately predict a patient's response to NACT are currently not available.

GOLPH3L (also referred to as GPP34R, GMx33 $\beta$ or FLJ10687) is a paralog of Golgi phosphoprotein 3 (GOLPH3) [4]. GOLPH3, which is located at chromosome $5 \mathrm{p} 13$, is a recently identified oncogene associated with various types of cancers, including lung cancer, gastric cancer, prostate cancer, breast cancer and colorectal cancer [5-9]. GOLPH3 is the first oncogene that is functionally related to Golgi trafficking and the maintenance of Golgi morphology [10]. Recent studies demonstrated that GOLPH3 plays a role in the Golgi dispersal process following DNA damage, and these findings have provided new insight into the cellular responses associated with standard cancer therapeutic agents [11]. However, compared with GOLPH3, little is known about GOLPH3L, and the functional role of GOLPH3L remains controversial. One paper reported that GOLPH3L is a putative inhibitor of GOLPH3, yet other studies reported that both GOLPH3 and GOLPH3L function as oncogenes [12-14].

This study, for the first time, investigated the clinical and functional significance of GOLPH3L in cervical squamous cell carcinoma. We evaluated the value of GOLPH3L in predicting cervical squamous cell carcinoma survival and NACT responsiveness. In addition, we silenced GOLPH3L expression in cervical cancer cell lines to assess the biological effects of GOLPH3L in cervical cancer. Finally, using phospho-antibody array experiments, we revealed a preliminary model of the changes in protein phosphorylation induced by GOLPH3L silencing.

\section{Materials and Methods}

\section{Patient selection, tissue collection and cell line information}

This study was approved by the medical ethics committee of Sun Yat-sen University Cancer. The 288 cervical squamous cell carcinoma patients selected for this study were primarily treated at Sun Yat-sen University Cancer Center between January 2002 and December 2012. Among the 288 patients, 130 were treated with NACT therapy. The criteria for patient selection were as follows: 1) patients with newly diagnosed cervical carcinoma with no history of previous treatment; 2) patients had not received preoperative radiotherapy; 3) the chemotherapy regimen for all 130 patients who were treated with NACT consisted of taxol, docetaxel or paclitaxel liposome plus cisplatin; and 4) patient tumor tissue was available in the archives of the Department of Pathology of Sun Yat-sen University Cancer Center. The tissue samples from the 158 patients who had not received NACT treatment were obtained during surgeries, and the samples from patients who had received NACT were obtained prior to NACT treatment. All tissue samples used for immunohistochemistry (IHC) analysis were paraffin-embedded. The 6 pairs of cervical carcinoma tissues and adjacent non-tumor tissues used for western blot and PCR experiments were stored at $-80^{\circ} \mathrm{C}$. The $\mathrm{SiHa}$ and HeLa cervical cancer cell lines were used in this study.

\section{IHC and evaluation of staining}

The tissue microarray (TMA) was constructed from small representative tissue samples from the 288 patients evaluated. IHC staining and interpretation of the staining results were performed according to a previously described protocol [14]. An anti-GOLPH3L antibody (ab121999, rabbit polyclonal, Abcam, USA) was used as the primary antibody. Two independent pathologists blinded to the clinicopathological information of the patients conducted the immunoreactivity scoring (IRS) of GOLPH3L expression. If the scores assigned by the 2 pathologists differed by a value greater than 3 , the specimens were rescored. The cutoff values for low and high expression were determined by the median IRS.

\section{Western blotting}

Western blot analysis was performed in 6 main steps: protein extraction, gel preparation, electrophoresis, electrotransfer, blocking and antibody incubation, and visualization. The specific protocol of the western blot experiments was previously described [14]. An antibody against GOLPH3L (ab121999, Rabbit polyclonal, Abcam, USA) was used as the primary antibody in the western blot experiments.

\section{Cell culture and Transient interference assay}

$\mathrm{SiHa}$ and HeLa cell lines were purchased from Type Culture Collection of the Chinese Academy of Sciences, Shanghai, China. The cells were cultured in DMEM medium with $10 \%(\mathrm{v} / \mathrm{v})$ fetal bovine serum in a $37^{\circ} \mathrm{C}$ humidified incubator with $5 \% \mathrm{CO}_{2}$. Small interfering RNA (siRNA) transfection was used to 
specifically silence GOLPH3L gene expression in $\mathrm{SiHa}$ and HeLa cells. Three pairs of GOLPH3L siRNA and negative control oligos were designed and synthesized by Shanghai GenePharma Co., Ltd. (Shanghai, China). The siRNA sequences are as follows: GOLPH3L-1: sense (5'-3') GGUGGGUAA AUGACCCUCAGCGUAU and antisense (5'-3') AUA CGCUGAGGGUCAUUUACCCACC; GOLPH3L-2: sense (5'-3') CGUCGCACUGAAAUAAGCAAGA ACU and antisense (5'-3') AGUUCUUGCUUAU UUCAGUGCGACG; GOLPH3L-3: sense (5'-3') GAUGGAAAGUGAGGAAGACAGUAAU and antisense (5'-3') AUUACUGUCUUCCUCACUU UCCAUC.

Transfections were conducted in 6-well plates. siRNA, Lipofectamine (TMRNAiMAX) and DMEM culture medium without serum were combined and added to the plates. After 6 hours of transfection, the culture medium was replaced with DMEM with $10 \%$ fetal bovine serum. Cells transfected with the negative control siRNA and the blank group were used as controls. Polymerase chain reaction (PCR) and western blot experiments were used to evaluate the efficiency of siRNA-mediated gene silencing after 48 hours of transfection.

\section{Reverse transcription-polymerase chain reaction (RT-PCR)}

Total RNA was extracted using TRIzol Reagent (Invitrogen, USA) according to the manufacturer's protocol. RNA concentration was measured using the NanoDrop spectrophotometer (ND-1000, Thermo Scientific, USA). Reverse transcription was conducted using the Promega reverse transcription kit. The resulting cDNA was used as a template for the RT-PCR reactions used to analyze the relative expression levels of GOLPH3L. The primer sequences were as follows: 5'-ATGACCCTCAGCGTAT GGAC-3' (F) and 5'-TTTGTCCCTTCCACTTCAGG-3' (R). Experiments with each cDNA sample were repeated a minimum of twice.

\section{Cell viability assay}

Cell viability was analyzed using the PrestoBlue assay (PrestoBlue ${ }^{\mathrm{TM}}$ Cell Viability Reagent, Invitrogen) according to the manufacturer's instructions. After transfecting cells with siRNA targeting GOLPH3L or the control sequences for 24, 48 or 72 hours, PrestoBlue reagent was added to the medium, and the cells were incubated for 30 minutes at $37^{\circ} \mathrm{C}$. We used the PrestoBlue assay to examine the effect of GOLPH3L (Hospira Australia Pty Ltd) on the sensitivity of $\mathrm{SiHa}$ and HeLa cells to cisplatin. Cells were excited at $560 \mathrm{~nm}$, and fluorescence was detected at $590 \mathrm{~nm}$. All experiments were repeated twice, and average values are plotted.

\section{Flow cytometry}

Cell apoptosis and cell cycle progression were evaluated using flow cytometry analysis of cells expressing GOLPH3L siRNA. Propidium iodide was used for the cell cycle assays, and propidium iodide with Annexin V-FITC was used for the apoptosis assays. Experiments were conducted using the cell apoptosis and cell cycle kits from KeyGen Biotech (Nanjing, China) according to the manufacturer's instructions.

\section{Phospho-protein profiling using a phospho-antibody array}

The phospho-antibody array was obtained from Full Moon BioSystems Inc. (Sunnyvale, CA, USA). Cell lysates obtained from the normal control SiHa cells and the GOLPH3 L-silenced SiHa cells were analyzed using the phospho-antibody microarray. The array consisted of 185 antibodies, with 6 replicates of each antibody imprinted on a standard size coated glass microscope slides. Briefly, $100 \mu \mathrm{g}$ of cell lysate in $100 \mu \mathrm{l}$ of reaction mixture were labeled with N,N-dimethylformamide to an ultra-titer of 10 $\mu \mathrm{g} / \mu \mathrm{l}$. Proteins from whole cell extracts were biotinylated at a 1:20 dilution, spotted on a microscope slide chamber with various antibodies bound to its surface and incubated at $4^{\circ} \mathrm{C}$ overnight. Cy3-streptavidin was subsequently added, and fluorescence was detected using a microarray scanner (GenePix 4000B, Axon Instruments). All experiments were performed according to the manufacturer's instructions. Positive and negative controls were used to normalize the results of the 2 membranes being compared. The fluorescence intensity of each array spot was quantified and used to calculate the mean value. The following equation was used to calculate the phosphorylation signal ratio of a given protein (protein A): phosphorylation ratio of $\mathrm{A}=$ phospho A/unphospho A. Phospho A represents the signal of the phosphorylated protein, and unphospho A represents the signal of the unphosphorylated protein. The phosphorylation ratio of proteins in the control SiHa cells and the GOLPH3L-silenced SiHa cells were compared, and proteins exhibiting a $>1.5$-fold phosphorylation ratio were considered significant. The Kyoto Encyclopedia of Genes and Genomes (KEGG) database (http:/ / www.genome.jp/ $\mathrm{kegg} /$ ) was utilized for data analysis and generation of the table.

\section{Statistical analysis}

Statistical analysis was performed using SPSS software (standard version 17.0, SPSS, Chicago, IL, USA) as previous described [14]. Graphical 
presentations were generated using the GraphPad Prism 4.0 software. A two-sided probability value less than 0.05 was considered statistically significant.

\section{Results}

\section{Patient characteristics}

In total, 288 cases of cervical squamous cell carcinoma were included in this study. All the patients received surgery (radical hysterectomy + pelvic lymph node \pm para-aortic lymph node sampling), and 130 of the patients received NACT prior to surgery. Patients ranged in age from 23 to 68 years with a median age of 45 . The clinical stage of all patients was determined according to the 2009 International Federation of Gynecology and Obstetrics (FIGO) staging system for cervical cancer. Among the 288 patients, 107 (37.2\%) were classified as stage IB1, $74(25.7 \%)$ as stage IB2, $31(10.8 \%)$ as stage IIA1, $53(18.4 \%)$ as stage IIA2 and the remaining 23 patients $(8 \%)$ were classified as stage IIB. The diameter of the cervical tumors ranged from 0.5 to 9.0 centimeters, with a median of 4.0 centimeters. Given that preoperative NACT might affect clinicopathological parameters, we excluded the 130 patients who received NACT treatment from the analysis of the association of GOLPH3L expression and clinicopathological parameters. We selected 150 patients who had initiated treatment prior to December 2008 for the survival analysis. The follow-up periods ranged from 1 to 139 months, with a median of 64 months. There were 31 cases of recurrent disease. The time until disease recurrence ranged from 3 to 54 months, with a median of 12 months.

The role of GOLPH3L in locally advanced cervical carcinoma was evaluated in 130 cervical cancer patients who received NACT therapy. The ages of these patients ranged from 29 to 66 years, with a median of 49 years. Sixty patients $(46.1 \%)$ were diagnosed with stage IB2 disease, 49 (37.7\%) were diagnosed with stage IIA2 disease and the remaining 21 patients $(16.2 \%)$ were diagnosed with stage IIB disease. The pretreatment SCC levels ranged from 0.3 to $70 \mu \mathrm{g} / \mathrm{L}$, with a median of $4.5 \mu \mathrm{g} / \mathrm{L}$. The pretreatment histological grade was classified as well differentiated for 2 patients, moderately differentiated for 51 and poorly differentiated for 77 patients. According to the pretreatment medical records, the diameter of the cervical tumors ranged from 2.5 to 9.0 centimeters, with a median of 5.0 centimeters. The particular NACT chemotherapy regimen and the number of cycles received were determined by the oncologist in charge. Ninety-eight patients received 2 cycles of NACT, and the remaining 32 patients received 3 cycles. In this cohort of 130 patients, 17 $(13.1 \%)$ achieved a pCR.

\section{GOLPH3L expression is an effective indicator of survival in cervical cancer patients}

We used 3 methods to evaluate the expression of GOLPH3L in cervical cancer samples. Real-time PCR and western blot experiments revealed that GOLPH3L mRNA and protein levels were elevated in all 6 cervical cancer samples compared with the matched adjacent non-cancerous tissues (Fig 1A, 1B).
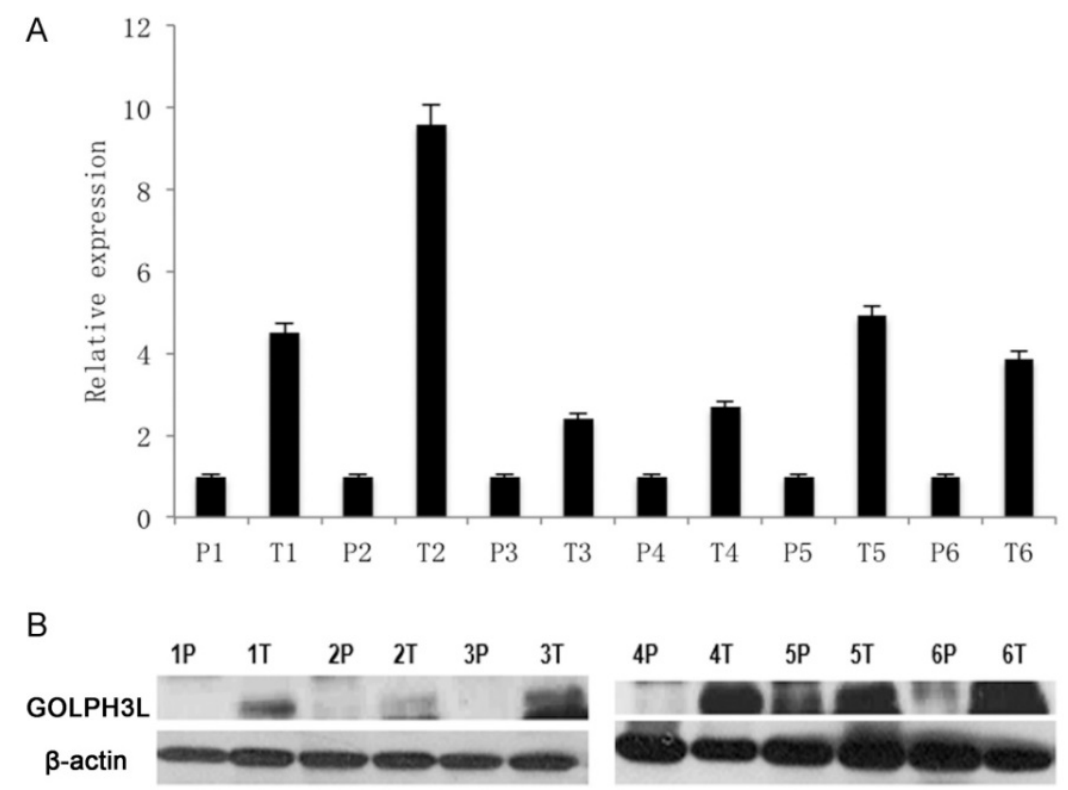

Figure 1. GOLPH3L mRNA and protein levels in cervical cancer samples. (1A)Real-time PCR showed that GOLPH3L mRNA and protein levels were elevated in all six cervical cancer samples when compared with their levels in adjacent non-cancerous tissues. (1B) Western blotting analysis showed that GOLPH3L mRNA and protein levels were elevated in all six cervical cancer samples when compared with their levels in adjacent non-cancerous tissues. 

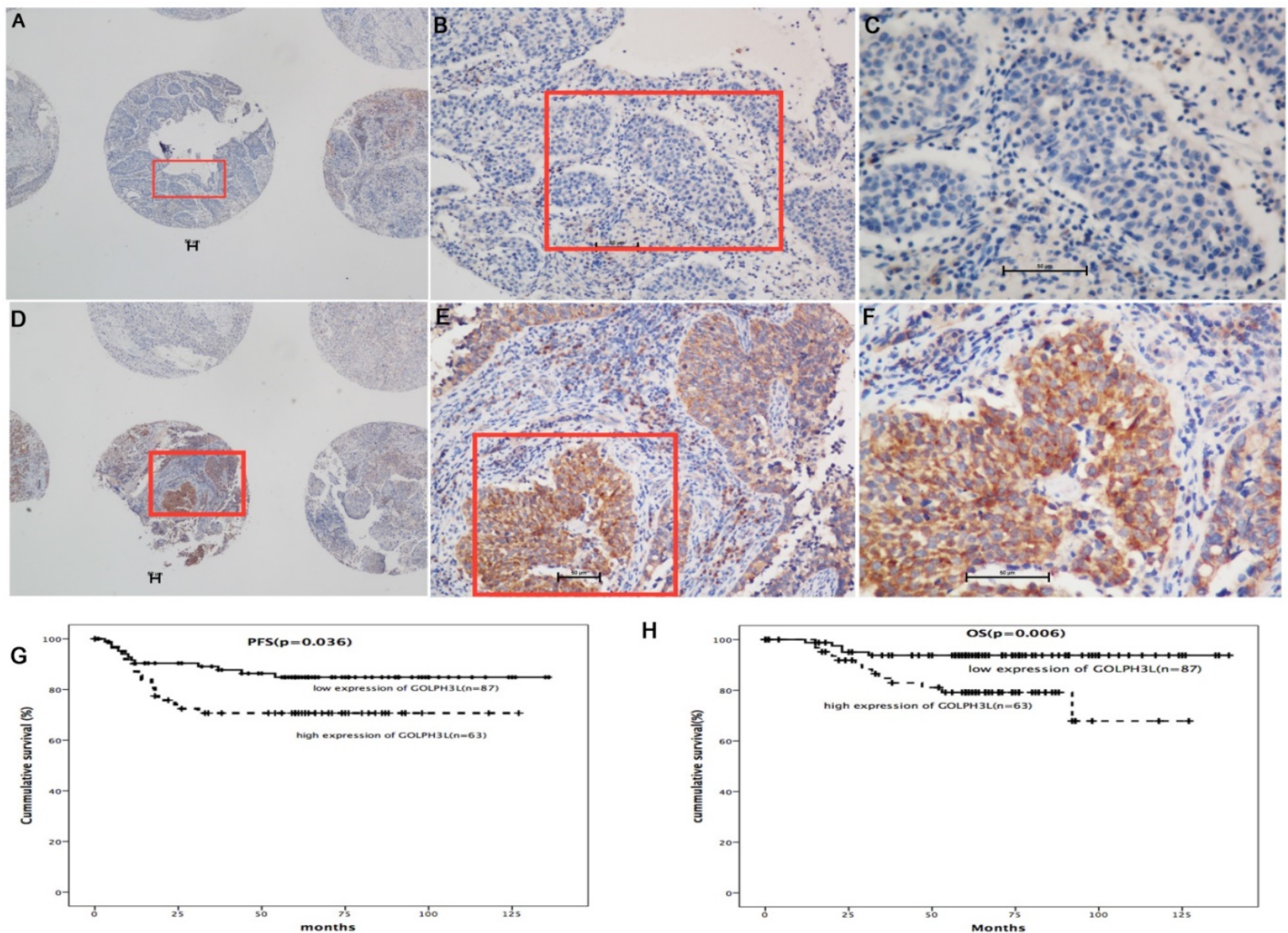

H

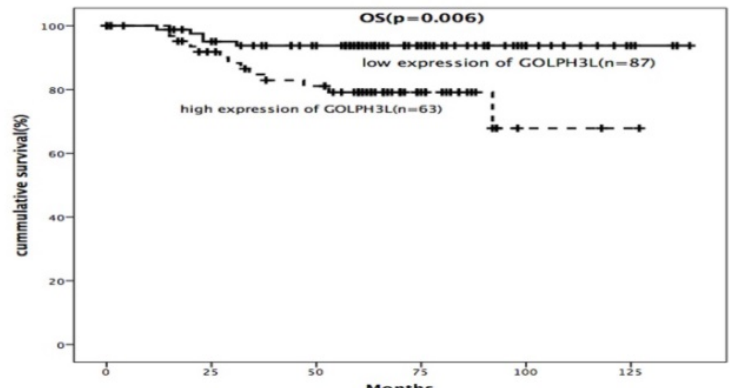

Figure 2. Immunohistochemistry and survival analysis in cervical cancer samples. In immunohistochemical analysis, immunoreactivity primarily appeared in the cytoplasm. High expression of GOLPH3L was observed in $119(41.3 \%)$ cervical cancer patients (Fig. 2 D, E and F), whereas low expression of GOLPH3L was observed in 169 (58.7\%) patients (Fig. 2 A, B and C). Patients with low expression of GOLPH3L had longer PFS and OS compared to those with high expression of GOLPH3L (Fig 2G, 2H).

GOLPH3L IHC staining was primarily observed in the cytoplasm (Fig 2A-2F). High expression of GOLPH3L were observed in 119 (41.3\%) cervical tissue samples, whereas low expression of GOLPH3L were observed in 169 (58.7\%) cervical tissue samples. The association between clinicopathological features and GOLPH3L expression are summarized in Table 1. The expression of GOLPH3L was significantly correlated with FIGO staging $(\mathrm{P}=0.033)$, cervical stromal invasion $(\mathrm{P}=0.037)$, cervical canal stromal invasion $(\mathrm{P}=0.027)$, lymph node metastasis $(\mathrm{P}=0.016)$ and positive surgical margins $(\mathrm{P}=0.015)$. No statistically significant associations were observed between GOLPH3L expression and age, histological grade, tumor diameter and lymphovascular space invasion (LVSI) $(\mathrm{P}=0.162,0.302,0.996$ and 0.287, respectively).

Among the 150 cases included in the survival analysis, GOLPH3L expression levels were defined as high in 87 cases and low in the remaining 63 cases. Patients with low expression levels of GOLPH3L demonstrated longer PFS and OS compared with patients expressing high levels of GOLPH3L (median
PFS of 83.5 months versus 54.9 months, $\mathrm{P}=0.036$; median OS of 131.7 months versus 102.8 months, $\mathrm{P}=0.006$, Fig $2 \mathrm{G}, 2 \mathrm{H}$ ).

The Kaplan-Meier survival analysis revealed that PFS was significantly associated with GOLPH3L expression $(\mathrm{P}=0.036)$, FIGO stage $(\mathrm{P}<0.001)$, cervical stromal invasion $(\mathrm{P}<0.001)$ and cervical canal stromal invasion $(\mathrm{P}=0.040)$. Cox's proportional hazard model demonstrated that cervical stromal invasion was the only independent prognostic indicator of PFS $(\mathrm{OR}=7.085,95 \%$ CI 2.143-23.422, $\mathrm{P}=0.001)$.

The Kaplan-Meier survival analysis demonstrated that OS was significantly associated with GOLPH3L expression $(\mathrm{P}=0.006)$, FIGO stage $(\mathrm{P}<0.001)$, cervical stromal invasion $(\mathrm{P}=0.001)$, cervical canal stromal invasion $(\mathrm{P}=0.005)$ and histological grade $(\mathrm{P}=0.015)$. The Cox proportional hazard model revealed that GOLPH3L expression (OR=3.231, 95\% CI 1.14-7.124, $\mathrm{P}=0.027)$, tumor differentiation $(\mathrm{OR}=2.013,95 \% \mathrm{CI} 1.044-3.881, \mathrm{P}=0.037)$ and cervical stromal invasion (OR=11.361, 95\% CI 1.502-85.963, $\mathrm{P}=0.019)$ were independent predictors of OS. 
Table 1. Associations of clinicopathological features with GOLPH3L expression.

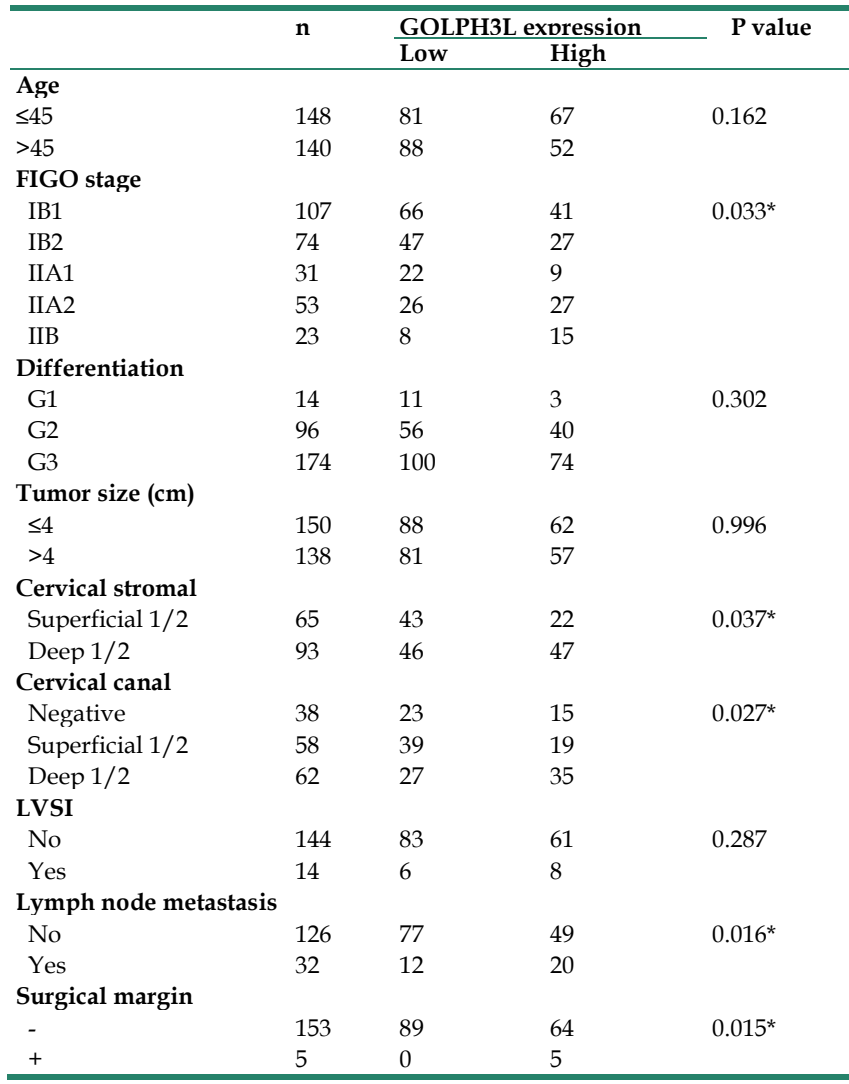

The value of GOLPH3 $L$ in the identification of NACT-responsive patients

In this study, we explored the value of GOLPH3L in predicting NACT-responsiveness in cervical squamous cell carcinoma patients. Western blot analysis revealed decreased GOLPH3L protein levels in patients who achieved pCR responses compared with those who failed (stable disease (SD) or progressive disease (PD)) to NACT (Fig 3A).

We also analyzed the association of GOLPH3L with NACT response in 130 patients with locally advanced cervical cancer with stage IB2, IIA2 or IIB disease. Among the patients with locally advanced cervical cancer who received NACT, the expression of GOLPH3L was significantly reduced in patients who achieved a pCR, suggesting that GOLPH3L might be associated with the response of cervical cancer patients to NACT. In addition, we compared the difference of $\mathrm{pCR}$ rates between patients with high and low expression levels of GOLPH3L. We found that patients with low levels of GOLPH3L expression were more responsive to NACT compared with patients expressing high levels of GOLPH3L (Table 2, $\mathrm{P}=0.032$ ). In addition, we analyzed the association between various clinicopathological features and parameters associated with NACT. Patients with low levels of GOLPH3L expression, poorer tumor differentiation, shorter NACT treatment intervals and smaller tumor sizes were more likely to achieve a pCR after NACT ( $\mathrm{P}=0.032,0.045,0.041,0.005$, respectively, Table 2).

Table 2. Associations of clinicopathological features and GOLPH3L expression with NACT response in patients with cervical cancer.

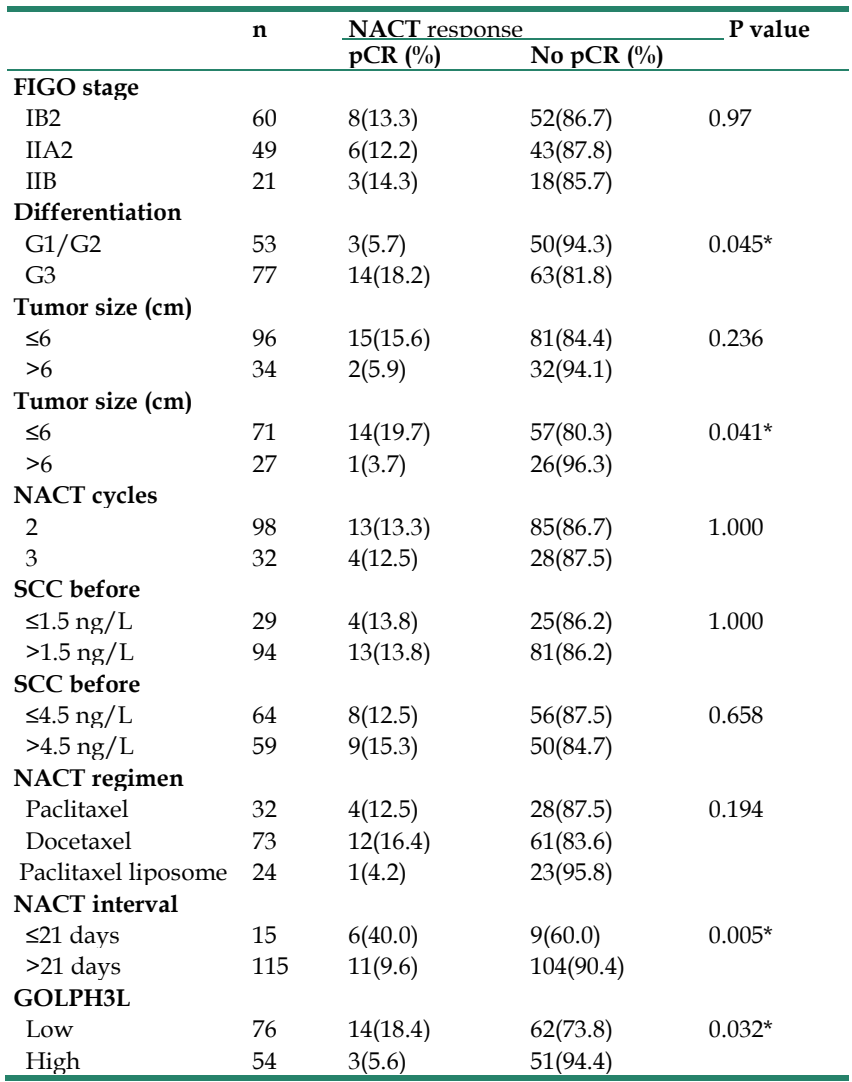

We further investigated whether GOLPH3L expression could be used to stratify locally advanced cervical carcinoma patients according to NACT sensitivity. Kaplan-Meier survival analysis demonstrated that PFS was significantly associated with GOLPH3L expression ( $\mathrm{P}=0.045)$, the number of NACT cycles received $(P=0.016)$, cervical canal stromal invasion $(\mathrm{P}=0.004)$ and retroperitoneal lymph node metastasis $(\mathrm{P}=0.001)$ (Table 3, Fig. 3B). COX analysis demonstrated that GOLPH3L expression was an independent prognostic indicator of PFS in patients with locally advanced cervical squamous cell carcinoma patients who received NACT (OR=9.918, 95\% CI 2.194-44.841, $\mathrm{P}=0.003)$. OS was significantly associated with GOLPH3L expression $(\mathrm{P}=0.01)$, FIGO stage $(\mathrm{P}=0.01)$, cervical canal stromal invasion $(\mathrm{P}=0.009), \mathrm{LVIS}(\mathrm{P}=0.048)$, retroperitoneal lymph node metastasis $(\mathrm{P}=0.001)$ and the number of NACT cycles 
$(\mathrm{P}=0.003)$ (Table 3, Fig. 3C). Cox proportional hazard model analysis revealed that retroperitoneal lymph node metastasis (OR=7.214, 95\% CI 1.927-27.010, $\mathrm{P}=0.003)$, the number of NACT cycles (1-2 cycles vs.
3-4 cycles, $\mathrm{OR}=4.991,95 \%$ CI $1.334-18.668, \mathrm{P}=0.017$ ) might be independent prognostic indicators of OS in patients with locally advanced cervical squamous cell carcinoma.

A

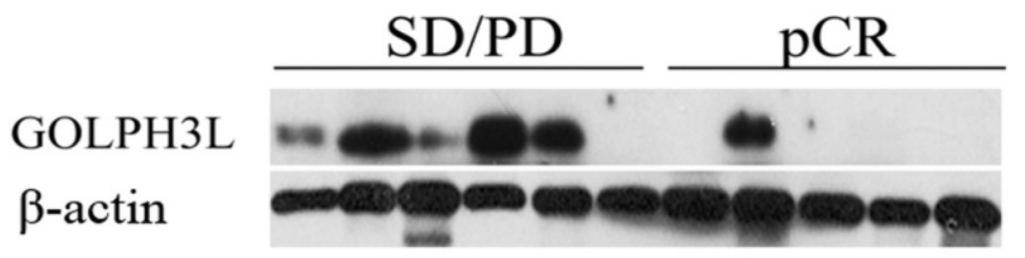

B

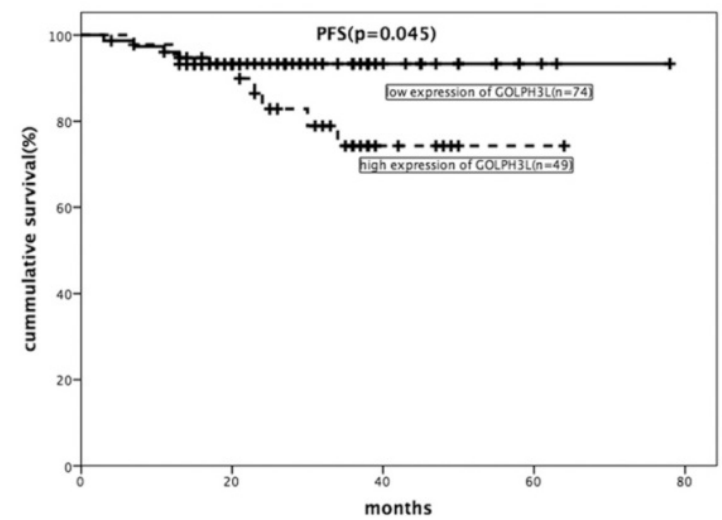

D

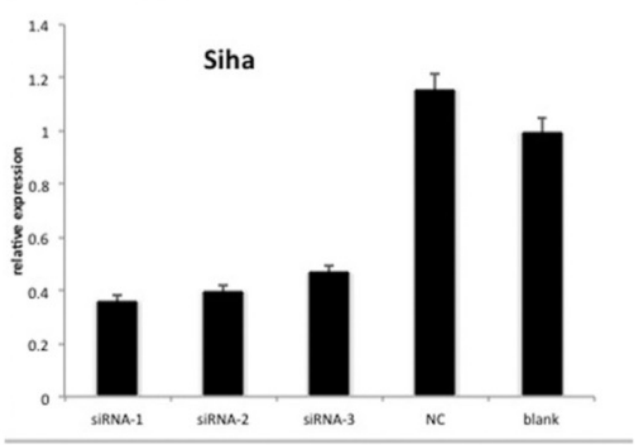

$\mathbf{F}$

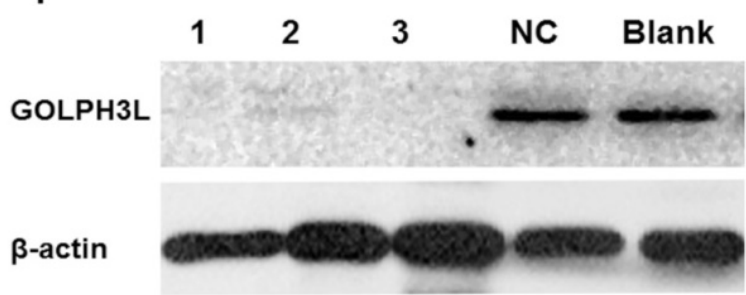

C

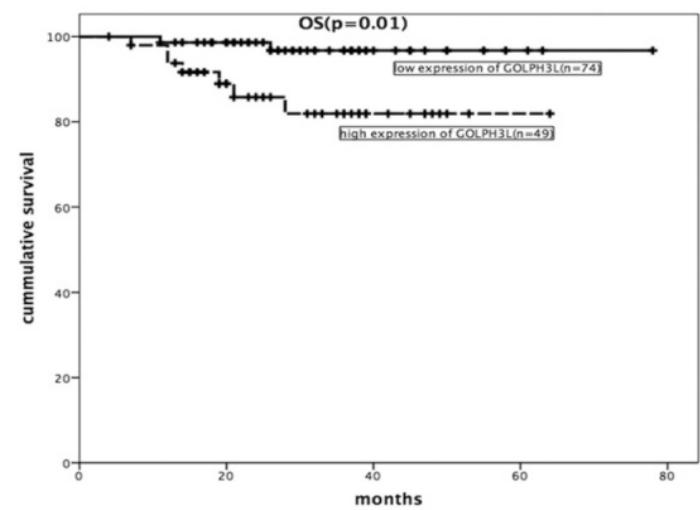

E

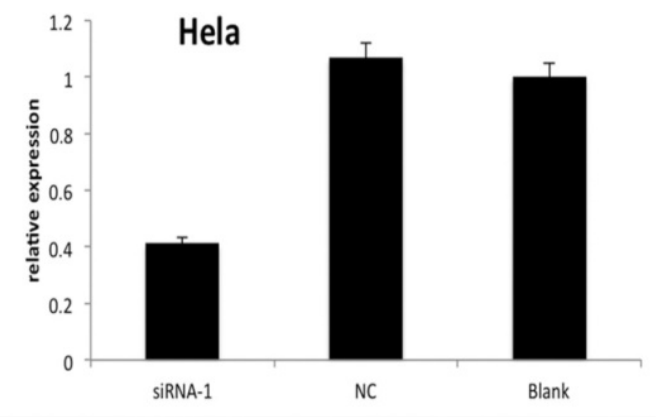

G

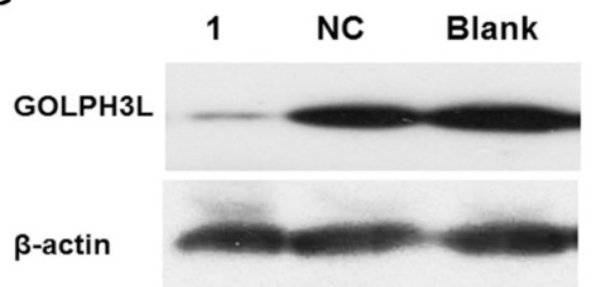

Figure 3. The value of GOLPH3 $L$ in the identification of NACT-responsive patients and SiRNA transfected cells. Western blotting analysis showed that GOLPH3L protein levels were decreased in patients who showed a PCR when compared with those showing no response (SD or PD) after NACT (Fig 3A). Patients with locally advanced cervical carcinoma who exhibited low expression of GOLPH3L had longer PFS and OS compared to those with high expression of GOLPH3L (Fig 3B, 3C). GOLPH3L RNA and protein expression levels were obviously reduced in the GOLPH3L siRNA groups when compared with the negative control and blank groups. Three different GOLPH3L siRNA sequences were transfected into SiHa cells, and it was found that all three siRNA sequences obviously reduced GOLPH3L RNA and protein expression levels in these cells (Fig 3D and 3F). Only one of the GOLPH3L siRNA sequences was chosen to transfect HeLa cells (Fig 3E and 3G). 
Table 3. Associations of clinicopathological features and GOLPH3L expression with prognosis of locally advanced cervical cancer.

\begin{tabular}{|c|c|c|c|c|c|}
\hline & $\mathbf{n}$ & PFS (months) $(95 \% \mathrm{CI})$ & P value & OS (months) $(95 \% \mathrm{CI})$ & P value \\
\hline \multicolumn{6}{|l|}{ FIGO stage } \\
\hline IB2+IIA2 & 103 & $71.6(67.6-75.6)$ & 0.119 & $75.2(72.5-77.9)$ & $0.01^{*}$ \\
\hline IIB & 20 & $42.4(35.8-48.9)$ & & $45.0(37.9-52.0)$ & \\
\hline \multicolumn{6}{|c|}{ Tumor size (cm) (by physical examination) } \\
\hline$\leq 6$ & 94 & $69.6(64.9-74.3)$ & 0.578 & $73.8(70.5-77.0)$ & 0.118 \\
\hline$>6$ & 29 & $42.6(39.5-45.7)$ & & $50.3(44.6-56.3)$ & \\
\hline \multicolumn{6}{|c|}{ Tumor size (cm) (by radiologic imaging) } \\
\hline$\leq 6$ & 65 & $45.2(42.1-48.3)$ & 0.411 & $49.0(46.0-52.0)$ & 0.891 \\
\hline$>6$ & 10 & $31.9(24.2-39.6)$ & & $35.3(30.3-40.3)$ & \\
\hline \multicolumn{6}{|l|}{ SCC } \\
\hline$\leq 4.5 \mathrm{ng} / \mathrm{L}$ & 58 & $59.2(55.3-63.2)$ & 0.222 & $62.2(58.2-64.3)$ & 0.341 \\
\hline$>4.5 \mathrm{ng} / \mathrm{L}$ & 58 & $50.4(45.5-55.3)$ & & $53.0(48.9-57.1)$ & \\
\hline \multicolumn{6}{|l|}{ Differentiation } \\
\hline G1-G2 & 49 & $71.7(65.8-77.6)$ & 0.423 & $73.6(68.8-78.4)$ & 0.618 \\
\hline G3 & 70 & $56.0(51.8-60.2)$ & & $58.4(54.8-61.9)$ & \\
\hline \multicolumn{6}{|c|}{ Cervical canal stromal invasion } \\
\hline Superficial $1 / 2$ & 69 & $71.5(66.8-76.4)$ & 0.376 & $74.1(71.1-78.4)$ & 0.145 \\
\hline Deep $1 / 2$ & 54 & $51.2(46.7-55.9)$ & & $52.7(48.7-56.6)$ & \\
\hline \multicolumn{6}{|c|}{ Cervical canal stromal invasion } \\
\hline Superficial 1/2 & 88 & $73.6(69.8-77.3)$ & $0.004^{*}$ & $75.4(73.0-78.3)$ & $0.009^{*}$ \\
\hline Deep $1 / 2$ & 35 & $46.7(39.8-53.5)$ & & $50.0(44.2-55.8)$ & \\
\hline \multicolumn{6}{|l|}{ LVSI } \\
\hline No & 106 & $71.0(66.9-75.1)$ & 0.152 & $73.9(70.7-77.1)$ & $0.048^{*}$ \\
\hline Yes & 17 & $49.3(40.4-58.2)$ & & $48.8(39.3-58.2)$ & \\
\hline \multicolumn{6}{|c|}{ Lymph node metastasis } \\
\hline No & 101 & $72.8(69.1-76.5)$ & $0.001^{*}$ & $75.5(72.6-78.2)$ & $0.001^{*}$ \\
\hline Yes & 22 & $41.5(32.5-50.5)$ & & $44.8(37.0-52.7)$ & \\
\hline \multicolumn{6}{|l|}{ Surgical margin } \\
\hline- & 114 & $70.1(66.0-74.3)$ & 0.889 & $72.9(69.4-76.3)$ & 0.571 \\
\hline+ & 9 & $51.9(40.6-63.2)$ & & $52.8(43.1-62.4)$ & \\
\hline \multicolumn{6}{|l|}{ NACT cycles } \\
\hline $1-2$ cycles & 94 & $72.6(68.8-76.8)$ & $0.016^{*}$ & $75.8(63.4-78.2)$ & $0.003^{*}$ \\
\hline 3-4 cycles & 29 & $45.9(37.7-54.6)$ & & $48.1(40.6-55.8)$ & \\
\hline \multicolumn{6}{|l|}{ NACT response } \\
\hline $\mathrm{pCR}$ & 17 & $48.1(44.4-51.7)$ & 0.436 & $48.0(44.3-51.7)$ & 0.801 \\
\hline No pCR & 106 & $53.4(46.9-59.9)$ & & $73.1(69.7-70.6)$ & \\
\hline \multicolumn{6}{|c|}{ GOLPH3L expression } \\
\hline Low & 74 & 73.5 (69.7-77.3) & $<0.05^{*}$ & 76.1 (73.5-78.7) & $0.01^{*}$ \\
\hline High & 49 & $94.1(81.3-107.0)$ & & 76.1 (73.5-78.7) & \\
\hline
\end{tabular}

\section{Effects of siRNA-mediated GOLPH3 L silencing in cervical cancer cell lines}

We evaluated RNA and protein extracted from cells that had been transfected for 48 hours using PCR and western blotting to verify the efficiency of siRNA-mediated GOLPH3L gene silencing. As shown in Fig. 3D-3G, GOLPH3L mRNA and protein levels were notably reduced in cells transfected with GOLPH3L siRNA compared with the negative control and blank groups. To investigate the effect of GOLPH3L knockdown in cervical cancer cells, we analyzed changes in cell growth using the Prestoblue assay. Transfection with GOLPH3L siRNA dramatically impaired cell growth in SiHa and HeLa cells $(\mathrm{P}<0.001)$ (Fig 4A, 4B). We also evaluated cell viability in siRNA-transfected cells treated with various concentrations of cisplatin. The inhibition of cell growth was significantly enhanced in GOLPH3L-silenced cells compared with control cells at all concentrations of cisplatin examined $(\mathrm{P}<0.05)$ (Fig 4C, 4D). We evaluated apoptosis and cell cycle changes in GOLPH3L-silenced SiHa and HeLa cells using flow cytometry. Compared with the negative control and blank groups, the proportion of GOLPH3L-silenced SiHa and HeLa cells in the G1 phase of the cell cycle significantly increased, indicating that silencing GOLPH3L expression induced cell cycle arrest at the G1 phase $(\mathrm{P}<0.05)$ (Fig. $4 \mathrm{E})$. Flow cytometry analysis revealed that the rate of apoptosis significantly increased in GOLPH3L-silenced cells compared with the negative control and blank cells $(\mathrm{P}<0.05)$ (Fig 4F). 


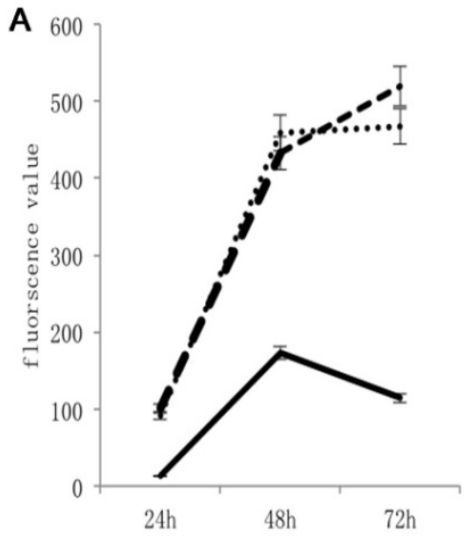

כ

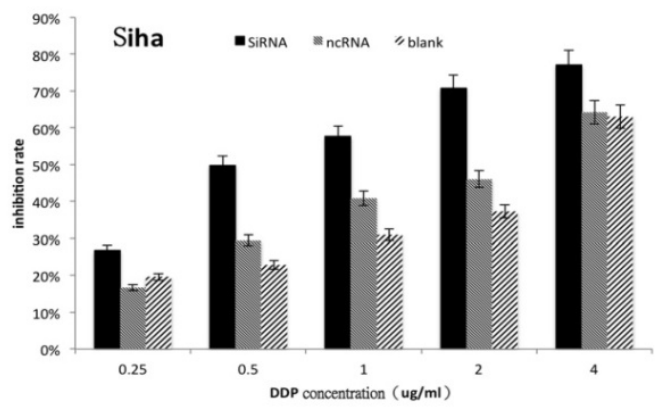

E

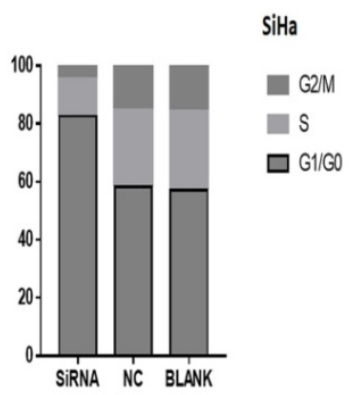

Siha

- SiRNA

- ncRNA -....blank transfecion

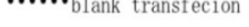

B

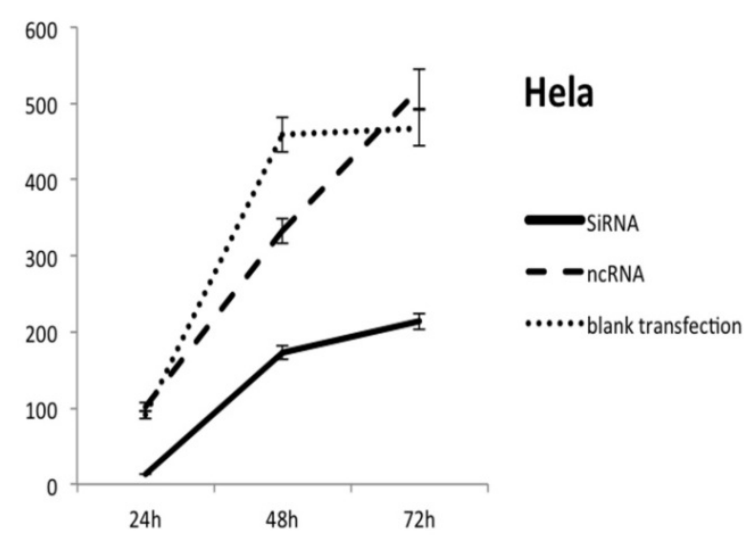

D

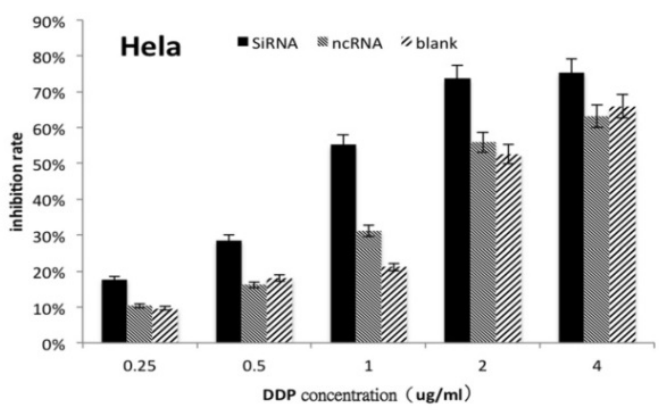

$\mathbf{F}$
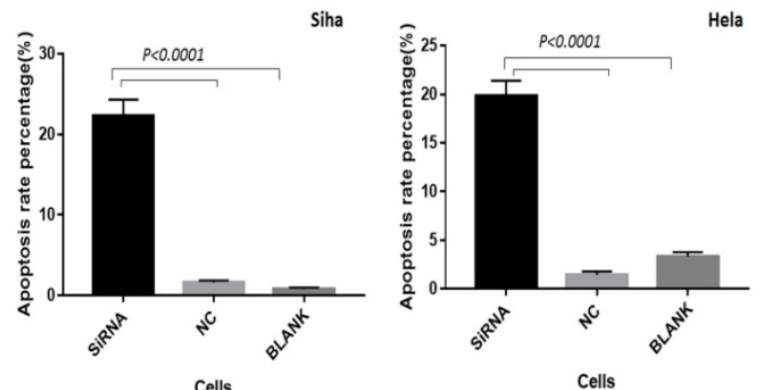

G

DNA damage or other stimuli (GOLPH3L SiRNA)
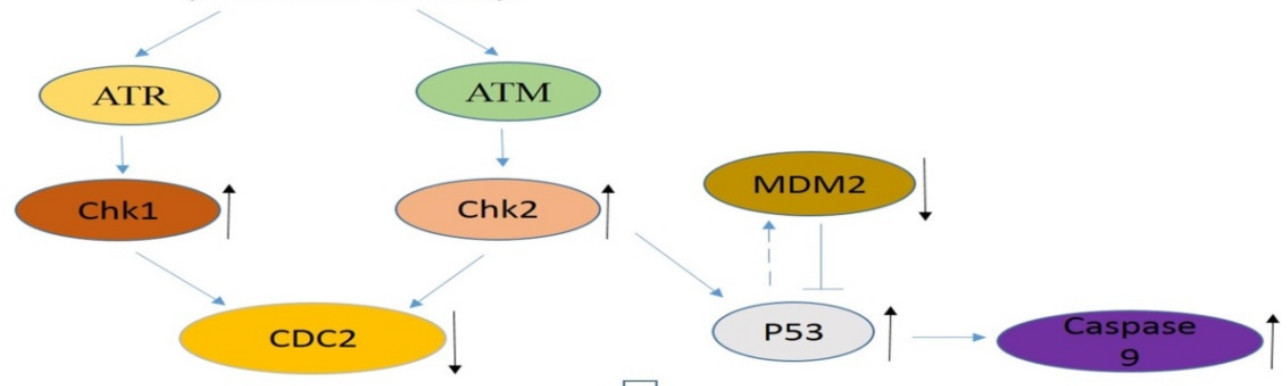

G1/S, G2/M checkpoints activation, cell cycle arrest, enhance chemotherapy response and apoptosis rate

Figure 4. Effects of siRNA-mediated GOLPH3 $\mathrm{L}$ silencing in cervical cancer cell lines and proposed model. (4A, 4B) GOLPH3L siRNA transfection dramatically impaired the growth of $\mathrm{SiHa}$ and HeLa cells. (4C, 4D) GOLPH3L-silenced cells were more sensitive to cisplatin treatment at each concentration tested. (4E) Compared to the negative control and blank control groups, the proportion of cells in Gl was increased significantly in the GOLPH3L-silenced groups, indicating Gl cell cycle arrest. (4F) Apoptosis rates were obviously increased in GOLPH3L-silenced cells compared with negative control and blank control cells. (4G) Using KEGG pathway analysis, we found that the activation and inactivation of these proteins are tightly associated in cell cycle regulation and apoptosis pathways. 
Table 4. Five critical proteins that were significantly phosphorylated or unphosphorylated in GOLPH3L-silenced $\mathrm{SiHa}$ cells.

\begin{tabular}{lllll}
\hline Name & $\begin{array}{l}\text { NC-phos } \\
\text { /unphos }\end{array}$ & $\begin{array}{l}\text { siRNA } \\
\text { phos/unphos }\end{array}$ & $\begin{array}{l}\text { siRNA } \\
\text { /NC }\end{array}$ & $\begin{array}{l}\text { Swiss } \\
\text { Prot }\end{array}$ \\
\hline CDC2 (Phospho-Tyr15) & 0.70 & 3.09 & 4.42 & P06493 \\
Caspase 9 (Phospho-Ser144) & 0.66 & 1.95 & 2.96 & P55211 \\
Chk2 (Phospho-Ser516) & 0.74 & 1.83 & 2.48 & O96017 \\
Chk1 (Phospho-Ser345) & 1.13 & 2.63 & 2.33 & O14757 \\
MDM2 (Phospho-Ser166) & 0.56 & 0.28 & 0.50 & Q00987 \\
\hline
\end{tabular}

Phospho-protein profiling using a
phospho-antibody array can detect the
phosphorylation status of proteins that might play key roles in the cellular responses to chemotherapy. This technique has the unique capability of quantitatively determining protein phosphorylation levels using antibodies against both the unphosphorylated and phosphorylated form of each protein. This approach can also be used to identify the signaling pathways potentially mediating the effects of GOLPH3L knockdown. We identified 5 proteins that exhibited significant changes in phosphorylation in GOLPH3L-silenced SiHa cells $(\mathrm{P}<0.01)$ (Table 4). In addition, using KEGG pathway analysis, we found that the activation status of the proteins identified is strongly associated with cell cycle regulation and apoptosis pathways (Fig 4G). Importantly, these proteins play key roles in cancer cell growth and response to chemotherapy.

\section{Discussion}

In the current study, we analyzed the expression pattern of GOLPH3L in tissue samples from 288 patients with cervical squamous cell carcinoma and evaluated the association between GOLPH3L expression and clinicopathological parameters and NACT sensitivity. Our data indicated that the expression level of GOLPH3L is inversely correlated with the prognosis of cervical squamous cell carcinoma patients and with the response to NACT in patients with locally advanced cervical squamous cell carcinoma. In addition, we examined the functional significance of GOLPH3L in cervical cancer cells. siRNA-mediated gene silencing of GOLPH3L significantly enhanced cell growth inhibition, apoptosis, cell cycle arrest, and cisplatin sensitivity. To identify the putative factors underlying the effects of GOLPH3L in cervical cancer cells, we used a phospho-antibody array to evaluate the phosphorylation status of proteins that play critical roles in cancer-associated processes.

GOLPH3, a paralog of GOLPH3L, is a conserved protein that localizes ubiquitously expressed at membrane of the Golgi apparatus. GOLPH3 was initially proposed to mediate protein trafficking between the Golgi apparatus and the endosomal system and to contribute to the maintenance of Golgi morphology $[15,16]$. Recently, GOLPH3 was also reported to be associated with multiple types of cancer, including lung cancer, gastric cancer, prostate cancer, breast cancer and colorectal cancer [5-9]. GOLPH3 plays an important role in the DNA damage response mediated by novel Golgi-associated pathway, and GOLPH3 overexpression confers resistance to DNA damaging agents, suggesting that GOLPH3 functions as an oncogene by mediating chemotherapy resistance [17, 18]. Based on these findings, it is reasonable to hypothesize that GOLPH3 might affect cancer prognosis by promoting resistance to DNA damaging therapeutic agents. The amino acid sequence of GOLPH3L is similar to GOLPH3; however, a previous study demonstrated that GOLPH3L functionally antagonizes GOLPH3 in the maintenance of Golgi morphology [12]. Another paper and our previously published paper reported contradictory data, demonstrating that GOLPH3L functions as an oncogene in rhabdomyosarcoma and epithelial ovarian cancer $[13,14]$.

We found that that GOLPH3L mRNA and protein levels were frequently elevated in cervical squamous cell carcinoma tissue samples compared with the corresponding adjacent non-cancerous tissues. GOLPH3L expression levels were strongly associated with FIGO staging, cervical stromal invasion, cervical canal stromal invasion, lymph node metastasis and positive surgical margins. Furthermore, GOLPH3L was an independent prognostic indicator of OS in cervical squamous cell carcinoma patients, consistent with observations from previous studies of GOLPH3L in rhabdomyosarcoma and epithelial ovarian cancer [13, 14]. To further confirm these findings, we transiently silenced the expression of GOLPH3L in cervical cancer cells and observed increased apoptosis, impaired cell proliferation and the induction of cell cycle arrest. Together, these data demonstrated that GOLPH3L plays an important role in the transformation of cervical cancer cells. As few studies have analyzed the association of GOLPH3L with cancer, the mechanism underlying these effects is unclear. However, we propose the following preliminary postulations. First, the results of flow cytometry and phospho-antibody profiling suggested that GOLPH3L strongly affects cell cycle regulation. In addition to the increased proportion of cells arrested in the G0/G1 phase of the cell cycle, the phospho-antibody array identified several key cell cycle-associated proteins, including CHK1, CHK2 and CDC2, which exhibited differential phosphorylation patterns in cells expressing 
GOLPH3L siRNA (Table 4). CHK1 and CHK2 are key mediators of cell cycle regulation, and the phosphorylation of $\mathrm{CHK} 1$ and CHK2 can activate the G1/S and G2/M checkpoints in cells with unrepaired DNA damage or can activate apoptosis [19, 20]. Additional experiments revealed that the phosphorylation of CDC2 and Caspase 9 were also significantly elevated in GOLPH3L-knockdown cells. $\mathrm{CDC} 2$ is the primary kinase regulating the M phase of the cell cycle, and the phosphorylation of CDC2 at tyrosine-15 inhibits CDC2 activity, thereby arresting cell cycle progression in the G2 phase [21]. In addition, GOLPH3L silencing increased the sensitivity of cervical cancer cells to chemotherapy. Together these findings provide new insights into the functional role of GOLPH3L. Many chemotherapeutic drugs exert their effects by inducing DNA damage. The observation that GOLPH3L silencing activated regulators of cell cycle checkpoints is likely to account for the increased sensitivity to chemotherapy. This is the first study to report that GOLPH3L affects cell cycle checkpoint proteins; however, the specific molecular mechanisms underlying this effect remain elusive. Additionally, the phospho-antibody array revealed that Caspase-9 is activated upon GOLPH3L silencing, consistent with the increase in apoptosis rates demonstrated by flow cytometry analysis.

In contrast to most other cancers, the etiology of cervical cancer is well characterized. Human papillomavirus accounts for most cases of cervical cancer [22]. In recent decades, the increased rates of cervical cancer screening in the United States and other countries have contributed to the decline of cervical cancer-associated death. However, if not diagnosed at an early stage, cervical cancer remains a life-threatening disease. Cervical cancer remains particularly fatal in the context of locally advanced cervical cancer, which is associated with an increased rate of recurrence and poorer survival rates compared with early-stage disease (stage IA, IB1 and IIA1) [2]. Previous studies have confirmed that NACT treatment might not only decrease parametrium invasion, lymph node metastasis and radiotherapy-induced damage but might also improve both OS and PFS in cervical cancer patients $[23,24]$. However, this does not guarantee that all patients will benefit from NACT treatment. The pCR rates associated with NACT is 10.3 to $19 \%$, and those patients who achieve $\mathrm{pCR}$ reap the maximum benefits from NACT [25]. Therefore, the identification of NACT-sensitive patients is critical for improving clinical outcomes. In this study, GOLPH3L was an effective clinical indicator of NACT sensitivity. Higher levels of GOLPH3L expression were observed in patients who responded poorly to NACT, and
GOLPH3L expression was an independent prognostic factor in patients who received NACT. These results indicate that the expression of GOLPH3L is inversely associated with NACT response. The cytotoxicity of cisplatin, a key component of NACT, is mediated by the formation of DNA crosslinks. GOLPH3L silencing reduces cell viability and increases the response of cervical cancer cells to cisplatin. Consistent with this observation, proteins associated with cell cycle arrest and cell cycle checkpoints were activated in GOLPH3L-silenced cells. In summary, this is the first study to report that GOLPH3L is associated with cell cycle regulation and cisplatin sensitivity. We sincerely hope that these data provide new insights into the association among GOLPH3L, cervical cancer and NACT response.

\section{Conclusion}

This study demonstrated that increased GOLPH3L expression levels are indicative of a poor response to chemotherapy and poor prognosis in cervical squamous cell carcinoma patients. We also demonstrated that GOLPH3L silencing is associated with a reduction in cell viability, induction of cell cycle arrest, and increased apoptosis rates and cisplatin sensitivity. Furthermore, proteins associated with cell cycle checkpoints and apoptosis were identified using the phospho-antibody array analysis. These findings potentially account for the clinicopathological significance and functional roles of GOLPH3L observed in cervical cancer. Together, these data indicate that additional studies evaluating the association between GOLPH3L and cervical cancer merit further investigation.

\section{Authors' contributions}

Yanling Feng and Shumei Yan conducted IHC and evaluation, as well as other experiments. Yanling Feng and Fan He designed the experiments and prepared the manuscript. He Huang, Qidan Huang and Ting Deng collected data, Huini Wu and Bei Gao analyzed data and created model, Jihong Liu supervised design of the study, data analysis and edited the manuscript.

\section{Competing interests}

The authors declare that no conflicts of interest exist.

\section{References}

1. Siegel RL, Miller KD, Jemal A. Cancer Statistics, 2015. CA Cancer J Clin. 2015;65:5-29.

2. Barbera L, Thomas G. Management of early and locally advanced cervical cancer. Semin Oncol. 2009;36:155-69.

3. Katsumata N, Yoshikawa H, Kobayashi H, Saito T, Kuzuya K, Nakanishi T, et al. Phase III randomised controlled trial of neoadjuvant chemotherapy plus radical surgery vs radical surgery alone for stages IB2, IIA2, and IIB cervical 
cancer: a Japan Clinical Oncology Group trial (JCOG 0102). Br J Cancer. 2013;108:1957-63.

4. Bell AW, Ward MA, Blackstock WP, Freeman HN, Choudhary JS, Lewis AP, et al. Proteomics characterization of abundant Golgi membrane proteins. J Biol Chem. 2001;276:5152-65.

5. Wang R, Ke ZF, Wang F, Zhang WH, Wang YF, Li SH, et al. GOLPH3 overexpression is closely correlated with poor prognosis in human non-small cell lung cancer and mediates its metastasis through upregulating MMP- 2 and MMP-9. Cell Physiol Biochem. 2015;35:969-82.

6. Hu BS, Hu H, Zhu CY, Gu YL, Li JP. Overexpression of GOLPH3 is associated with poor clinical outcome in gastric cancer. Tumour Biol. 2013;34:515-20.

7. Li W, Guo F, Gu M, Wang G, He X, Zhou J, et al. Increased expression of GOLPH3 is associated with the proliferation of prostate cancer. J Cancer. 2015;6:420-9.

8. Zeng Z, Lin H, Zhao $\mathrm{X}$, Liu G, Wang $\mathrm{X}, \mathrm{Xu}$ R, et al. Overexpression of GOLPH3 promotes proliferation and tumorigenicity in breast cancer via suppression of the FOXO1 transcription factor. Clin Cancer Res. 2012;18:4059-69.

9. Wang Z, Jiang B, Chen L, Di J, Cui M, Liu M, et al. GOLPH3 predicts survival of colorectal cancer patients treated with 5-fluorouracil-based adjuvant chemotherapy. J Transl Med. 2014;12:15.

10. Scott KL, Kabbarah O, Liang MC, Ivanova E, Anagnostou V, Wu J, et al. GOLPH3 modulates mTOR signalling and rapamycin sensitivity in cancer. Nature. 2009;459:1085-90.

11. Farber-Katz SE, Dippold HC, Buschman MD, Peterman MC, Xing M, Noakes $\mathrm{CJ}$, et al. DNA damage triggers Golgi dispersal via DNA-PK and GOLPH3. Cell. 2014;156:413-27.

12. Ng MM, Dippold HC, Buschman MD, Noakes CJ, Field SJ. GOLPH3L antagonizes GOLPH3 to determine Golgi morphology. Mol Biol Cell. 2013;24:796-808.

13. Kunigou O, Nagao H, Kawabata N, Ishidou Y, Nagano S, Maeda S, et al. Role of GOLPH3 and GOLPH3L in the proliferation of human rhabdomyosarcoma. Oncol Rep. 2011;26:1337-42.

14. Feng $\mathrm{Y}$, He $\mathrm{F}, \mathrm{Wu} \mathrm{H}$, Huang $\mathrm{H}$, Zhang $\mathrm{L}$, Han $\mathrm{X}$, et al. GOLPH3L is a Novel Prognostic Biomarker for Epithelial Ovarian Cancer. J Cancer. 2015;6:893-900.

15. Dippold HC, Ng MM, Farber-Katz SE, Lee SK, Kerr ML, Peterman MC, et al. GOLPH3 bridges phosphatidylinositol-4- phosphate and actomyosin to stretch and shape the Golgi to promote budding. Cell. 2009;139:337-51.

16. Wood CS, Schmitz KR, Bessman NJ, Setty TG, Ferguson KM, Burd CG. PtdIns4P recognition by Vps74/GOLPH3 links PtdIns 4-kinase signaling to retrograde Golgi trafficking. J Cell Biol. 2009;187:967-75.

17. Buschman MD, Rahajeng J, Field SJ. GOLPH3 links the Golgi, DNA damage, and cancer. Cancer Res. 2015;75:624-7.

18. Buschman MD, Xing M, Field SJ. The GOLPH3 pathway regulates Golgi shape and function and is activated by DNA damage. Front Neurosci. 2015;9:362

19. Bartek J, Lukas J. Chk1 and Chk2 kinases in checkpoint control and cancer. Cancer Cell. 2003;3:421-9.

20. Chen Y, Poon RY. The multiple checkpoint functions of CHK1 and CHK2 in maintenance of genome stability. Front Biosci. 2008;13:5016-29.

21. Gu Y, Rosenblatt J, Morgan DO. Cell cycle regulation of CDK2 activity by phosphorylation of Thr160 and Tyr15. EMBO J. 1992;11:3995-4005.

22. Walboomers JM, Jacobs MV, Manos MM, Bosch FX, Kummer JA, Shah KV, et al. Human papillomavirus is a necessary cause of invasive cervical cancer worldwide. J Pathol. 1999;189:12-9.

23. Rydzewska L, Tierney J, Vale CL, Symonds PR. Neoadjuvant chemotherapy plus surgery versus surgery for cervical cancer. Cochrane Database Syst Rev. 2012;12:CD007406

24. Kim HS, Sardi JE, Katsumata N, Ryu HS, Nam JH, Chung HH, et al. Efficacy of neoadjuvant chemotherapy in patients with FIGO stage IB1 to IIA cervical cancer: an international collaborative meta-analysis. Eur J Surg Oncol. 2013;39:115-24.

25. Hu T, Li S, Chen Y, Shen J, Li X, Huang K, et al. Matched-case comparison of neoadjuvant chemotherapy in patients with FIGO stage IB1-IIB cervical cancer to establish selection criteria. Eur J Cancer. 2012;48:2353-60. 